\title{
Tipos de aguas minero-medicinales en yacimientos arqueológicos de la península Ibérica
}

\author{
Lucía Moltó *
}

\section{INTRODUCCIÓN}

Las aguas minero-medicinales utilizadas en la Península Ibérica durante la Antigüedad son poco conocidas, pues más bien los estudios se han dirigido a los restos arqueológicos y/o a su posible significado religioso. Es por esto, por lo que se ha considerado importante estudiar los diferentes tipos de aguas minero-medicinales que correspondía a yacimientos arqueológicos relacionados en balnearios de uso actual o en desuso, con el objeto de aportar una aproximación inicial que nos permita conocer más científicamente por qué propiedades curativas fueron utilizadas estas aguas en la Antigüedad.

Para ello, se han tenido en cuenta los yacimientos arqueológicos de la Península lbérica relacionados con aguas minero-medicinales, incluyendo aquellos manantiales en los que existe algún tipo de documentación arqueológica o epigráfica que indica actividades de tipo termal. Algunos no se han podido documentar por falta de fiabilidad en los datos conocidos o bien, por ausencia de análisis de sus aguas.

El catálogo de estas aguas, aunque debe considerarse incompleto hasta que nueva documentación lo vaya enriqueciendo, se ha considerado válido, ya que constituye una muestra suficientemente representativa de los diversos tipos de aguas minero-medicinales.

En este trabajo se ha procedido, en primer lugar, a clasificar los yacimientos según las características y propiedades de sus aguas, así como

* Médico-especialista en Hidrologia Médica. 
de sus temperaturas. Luego, hemos determinado las posibles indicaciones terapéuticas de dichos tipos de aguas para deducir si su posible aplicación curativa en la Antigüedad tenía o no base científica. Este procedimiento, a pesar de sus limitaciones, ha permitido obtener algunos resultados objetivos y de indudable interés que, teóricamente, abren nuevas perspectivas en este campo de estudios.

\section{CARACTERISTICAS MINERO-MEDICINALES DE LAS AGUAS EN LOS YACIMIENTOS DE LA PENÍNSULA IBÉRICA}

En este trabajo se debe tener en cuenta que los yacimientos arqueológicos documentados asociados a aguas en la Península Ibérica han sido 52 (Apéndice l), cantidad infima si se compara con la de otros paises limítrofes, lo que da idea del atraso en este tipo de estudios. Llama la atención que el número de yacimientos documentados en la Península Ibérica asociados a fuentes y creencias salutíferas es muy reducido comparado con los muy numerosos casos de otros países vecinos, especialmente de las Galias '. La causa no parece que se deba interpretar sólo como consecuencia de falta de investigación en este campo ${ }^{2}$, sino que también pudiera reflejar diferencias culturales, especialmente un mayor arraigo en dichas regiones de creencias religiosas y rituales relacionadas con las aguas, particularmente frecuentes en el mundo céltico ${ }^{3}$, tema en el que no es posible entrar aquí.

La clasificación de las aguas según sus características permite determinar su finalidad curativa. Existen diversas clasificaciones, pero noso-

${ }^{1}$ Resulta sumamente reveladora la comparación de la situación de la Península Ibérica con la de las vecinas Galias. Véase, por ejemplo, la Tesis Doctoral de P. Audin (1978), donde se recogen hasta 829 fuentes sagradas sólo en los Departamentos del NW de Francia, con una densidad media de $1 / 141 \mathrm{~km}^{2}$, que oscilan desde $1 / 572 \mathrm{~km}^{2}$ en Mayenne hasta alcanzar la de $1 / 63 \mathrm{~km}^{2}$, en la de Finisterre.

Véase, a nivel más general, las recopilaciones y otros trabajos como los de Audin, $P$ 1983; Bourgols, C. 1986; Bullot, J.-G. 1867; Duval, P.-M. 1989: 1172; Esperandieu, E. 1912; Grenier, A. 1960; Thevenot 1966; id. 1968; Pelletier, A. 1985; Pilule, A. y M. 1985; Valleat, C. 1932, etc.

${ }^{2}$ Mora, G. 1981; Díez de Velasco, F. P. 1985.

${ }^{3}$ Blázquez, J. M. 1977; Garcia, B. 1986; Eliade, M. 1969: 30 s.; Vries, J. de 1975: 79 s.; Duval, J.-P. 1976: 76-77; Lambrecht, P. 1953; Maringer, J. 1973; Markale, J. 1969: 19 s. y 24; Le Roux, F.-Guyonvare'H, Chr. J. 1982: 98, 103 y 122; Thevenot, E. 1954; id. 1968; Valllat, Cl. 1932, etc. 
Tipos de aguas minero-medicinales ...

tros hemos seguido el método actual que sigue la Escuela de Hidrología Médica en España ${ }^{4}$, de la Universidad Complutense de Madrid, basado tanto en su contenido aniónico como catiónico.

También se debe tener presente la complejidad que ofrecen la composición de la mayoría de las aguas, hecho que se refleja en la dificultad de su clasificación, ya que a su componente predominante, generalmente el más tenido en cuenta para su clasificación dentro de un tipo u otro, frecuentemente se asocian otros elementos y características que también pueden aportar importancia curativa, lo que explica las variaciones que existen en la clasificación de las aguas minero-medicinales, como consecuencia de los distintos procedimientos ideados para llegar a clasificar un conjunto de acusadas características polimorfas ${ }^{5}$.

Esto es esencial tenerlo en cuenta para llegar a comprender la diversidad de efectos curativos que puede ofrecer un agua determinada. Por ello es complejo el problema de interpretar sus propiedades médicas, esencial para este tipo de estudios ${ }^{6}$. En este sentido se ha procedido, en primer lugar, a agrupar las aguas de características afines $y$, a continuación, se han valorado las opiniones más solventes y generalizadas sobre sus indicaciones, recogidas tanto de la bibliografía actual como, en caso de estar la fuente termal en desuso, recurriendo a bibliografía contemporánea a su uso. Asi pues clasificamos las aguas en tres grupos:

I. Aguas minerales que contienen más de $1 \mathrm{~g} / \mathrm{l}$ de sustancias mineralizantes. A este grupo pertenecen:

Aguas cloruradas.-Este tipo de aguas son tónicas, estimulantes y reparadoras de la cicatrización, dando buenos resultados en su actuación sobre la piel y sobre mucosas, destacándose también su uso en el aparato respiratorio y afecciones ginecológicas, como también en neuralgias y afecciones quirúrgicas traumáticas.

Las aguas cloruradas se clasifican, a su vez, en:

- Cloruradas fuertes (más de $50 \mathrm{gr} / \mathrm{l}$ ), no se ha documentado ninguna.

\footnotetext{
${ }^{4}$ Armiso, M. de 1967: 124.

${ }^{5}$ SAN ROMÁ, J. de (1945), ya hace referencia al problema de la clasificación de las aguas, ya que un mismo yacimiento puede incluirse en dos o más grupos de agua.

${ }^{6}$ San Román, J. 1945; Armijo, M. de 1967: 125.
} 
- Cloruradas medianas (de 10 a $50 \mathrm{gr} / \mathrm{l}$ ), tampoco se ha documentado ninguna.

- Cloruradas débiles (de menos de $10 \mathrm{gr} / \mathrm{l}$ ). En los yacimientos arqueológicos peninsulares si son frecuentes las cloruradas débiles, entre las que cabe considerar las de Caldas de Montbuy, Fitero, Archena y Ontaneda.

Aguas sulfatadas.-Son descongestionantes y purgantes y son utilizadas principalmente en bebida.

Las aguas sulfatadas se pueden clasificar en:

- Sulfatadas sódicas: Baños de Cerrato y Linares.

- Sulfatadas magnésicas: Cerro de los Santos.

- Sulfatadas mixtas: Vilavella.

No se documentan sulfatadas cálcicas.

Aguas bicarbonatadas. - Las aguas bicarbonatadas tienen sus principales indicaciones en las enfermedades del aparato digestivo. Son antidispépticas y antiácidas. Su principal forma de administración es en bebida.

Las aguas bicarbonatadas se clasifican en:

- Bicarbonatadas sódicas: Baños de Junquera de Ambia, Ben Saude, Boñar, Caldas de Malavella, Caldas de Monchique, Caldelas y Mondariz.

- Bicarbonatadas cálcicas: Alhama de Aragón, Chaves y Senhora da Luz.

No existe ninguno documentado de aguas bicarbonatadas mixtas.

Aguas carbogaseosas o carbónicas.-Son aguas que contienen gas ácido carbónico libre. Tienen un sabor vivo picante y se utilizan como aguas de mesa, aunque también puede utilizarse en forma de baños para afecciones del aparato circulatorio: hipertensiones, vasculopatías, etc.

Este tipo de aguas no son frecuentes en los yacimientos hispánicos, salvo el caso representativo de Mondariz.

II. Aguas minerales caracterizadas por contener elementos especiales. A este grupo pertenecen: 
Aguas sulfuradas.-Este tipo de aguas se distingue fácilmente por su característico olor fétido parecido a huevos podridos. En su mayor parte suelen ser hipertermales, aunque las hay también frias.

Son antialérgicas, antiinflamatorias y antireumáticas. Dirigen su acción especialmente hacia la piel, por lo que sus aplicaciones principales son en enfermedades cutáneas, como dermatosis, etc. También se utilizan en afecciones de vias respiratorias, como rinitis, laringitis, bronquitis, etc., por medio de inhalaciones. Igualmente son útiles en procesos reumáticos y del aparato locomotor.

Las aguas sulfuradas se clasifican en:

- Sulfuradas sódicas: C. de Cuntis, Baños de Bande, Baños de Ledesma, Baños de Montemayor, Burgas, Caldas de Canaveses, Caldas das Taipas, Caldas de Vizela, Fadagosa, Guitiriz, Lugo, Lisboa, Retortillo, El Salugral, San Gregorio de Brozas, San Vicente, Santa Eulalia de Caldelas, São Pedro do Sul, Tiermas, Torreblanca.

- Sulfuradas cálcicas: Carballo.

- Sulfuradas cloruradas: Archena, Caldas de Reyes y Lisboa.

Aguas ferruginosas.-Estas aguas contienen como elemento especial el hierro. La mayor parte de ellas son frías. Son estimulantes de la nutrición, antianémicas y reconstituyentes, por lo que sus principales indicaciones son para procesos que cursan con déficit de hierro, transtornos del desarrollo, convalescencias y enfermedades cuyo principal elemento sea la debilidad. Por ello se usan como bebida principalmente.

Aguas ferruginosas en la Península Ibérica sólo se han encontrado hasta ahora en Saelices y Santa Cruz de Loyo.

Aguas radioactivas. - Son aguas que contienen radioactividad natural. Son calmantes, analgésicas, antiálgicas y descongestionantes.

En consecuencia, sus aplicaciones son muy diversas, pero principalmente en enfermedades articulares y reumáticas, procesos asmáticos y afecciones circulatorias.

Las principales aguas radioactivas documentadas en yacimientos de la Península Ibérica son las de Alange, Alhama de Aragón, Boñar, Caldas das Taipas, Caldas de Canaveses, Caldas de Montbuy, Caldas de Reyes, Caldas de Vizela, Caldelas, Fitero, Guitiriz, Ledesma, Molgas, Montemayor, Panticosa, Retortillo, San Vicente Pinheiro, San Pedro do Sul.

III. Aguas de baja mineralización u oligometálicas: 
Aguas oligominerales.-Se caracterizan porque su mineralización es inferior a $1 \mathrm{~g} / \mathrm{l}$ y la aplicación de estas aguas es diversa según sean termales o frías. Las termales o acratotermas (mayores de $20^{\circ} \mathrm{C}$ ) son, generalmente, de uso externo en forma de baños debido a su acción sedante y analgésica, mientras que las frías o acretopegas (menores de $20^{\circ} \mathrm{C}$ ) se utilizan primordialmente en bebida, siendo su principal acción la diurética, pues aumentan la eliminación urinaria y tienen efecto de arrastre, por lo que se emplean, dado sus efectos diuréticos, para litiasis urinarias, gota y alteraciones funcionales de las vias excretoras.

Las aguas oligominerales documentadas en yacimientos arqueológicos son las siguientes: Alange, Alhama de Aragón, Boñar, Caldas de Reyes, Caldelas, La Garriga, Molgas, Panticosa, Vilavella.

\section{TEMPERATURAS DE LAS AGUAS MINERO-MEDICINALES}

Tiene indudable interés para el conocimiento del uso de las aguas el clasificarlas según su temperatura, ya que dependiendo de ésta son diversos sus efectos sobre el organismo.

Debemos decir que las aguas calientes son relajantes, sedantes y combaten la fatiga muscular, mientras que las aguas frías, por el contrario, son tónicas y estimulantes y se suelen utilizar, como ya hemos dicho anteriormente, más bien para bebida.

Hemos clasificado las aguas de los yacimientos termales en tres grupos, aunque algunos yacimientos termales están representados en más de uno por tener manantiales de diferente temperatura.

Aguas calientes (de más de $36{ }^{\circ} \mathrm{C}$ ): Alhama de Aragón, Archena, Baños de Bande, Baños de Río Caldo, Burgas, Caldas de Cuntis, Caldas de Malavella, Caldas de Montbuy, Caldas de Reyes, Caldas de Vizela, Caldelas, Chaves, Fitero, Ledesma, Lugo, Molgas, Montemayor, Lisboa, Retortillo, Panticosa, San Pedro do Sul, Vilavella.

Aguas templadas (de 30 a $36{ }^{\circ} \mathrm{C}$ ): Alange, Alhama de Aragón, Boñar, Caldas de Canaveses, Caldas de Monchique, Caldas de Montbuy, Caldas de Reyes, Caldas de Vizela, Caldelas, Carballo, Ledesma, Linares, Lugo, Lisboa, Panticosa, Tiermas, Vilavella.

Aguas frias (menos de $30^{\circ} \mathrm{C}$ ): Baños de Junquera de Ambia, Caldas de Cuntis, Guitiriz, Mondariz, Saelices, San Gregorio de Brozas, San Vicente Pinheiro, Torreblanca. 
Tipos de aguas minero-medicinales ...



Fig. 1. Cuadro de temperaturas de las aguas minero-medicinales de la Peninsula Ibérica (línea de trazos) comparadas a las asociadas a yacimientos arqueológicos (línea continua). Las cifras sobre dicha linea indican el \% (arriba) y la proporción (debajo) de las aguas arqueológicamente documentadas en cada grupo de temperaturas, lo que evidencia una clara preferencia por las aguas hipertermales. 
La temperatura mayor de las conocidas corresponde a Caldas de Chaves con $73,5^{\circ} \mathrm{C}$, y las más fria a Mondariz, con $13{ }^{\circ} \mathrm{C}$.

USO DE LOS DIVERSOS TIPOS DE AGUAS MINERO-MEDICINALES

El análisis de las aguas minero-medicinales y la clasificación de sus yacimientos respectivos en grupos según los tipos de agua documentados ofrece gran interés. Aunque no se pueda considerar completa, dicha clasificación proporciona una idea suficientemente válida del conjunto de yacimientos termales utilizados con finalidad salutífera en la Península lbérica en la Antigüedad.

En consecuencia, estos resultados y los consiguientes agrupamientos en los diferentes tipos de aguas pueden ser representativos de las aguas minero-medicinales que se utilizaban en la Antigüedad y pueden servir como muestra para ser contrastada con el conjunto de las aguas mineromedicinales de la Península Ibérica, que se recogen en el correspondiente cuadro de conjunto basado en alguna de las diversas recopilaciones existentes de aguas minero-medicinales ${ }^{7}$.

En efecto, si se parte de la hipótesis de que la asociación entre un yacimiento arqueológico y una fuente termal permite documentar el aprovechamiento de las cualidades salutíferas de ésta, la muestra recogida puede considerarse suficientemente representativa para abordar una aproximación a este campo de la Historia del Termalismo y de la Historia de la Medicina en general, tema hasta ahora nunca planteado en un análisis de conjunto.

Así pues, diremos que se han identificado en la Península Ibérica más de 50 yacimientos arqueológicos asociados a aguas minero-medicinales, de los cuales prácticamente en todos se han podido identificar las características y propiedades minero-medicinales de sus aguas.

El primer hecho a destacar es que todas las aguas documentadas asociadas a elementos arqueológicos son de tipo minero-medicinal, pues no existe ninguna carente de propiedades curativas, hecho señalado en alguna ocasión, por ejemplo, en la fuente del santuario galo-romano de

${ }^{7}$ Pérez de la Flor, J. 1851: cuadro final; Garcia Lopez, A. 1876: 126-133; San Román, J. de 1945: 217 s.; Armijo, M. de 1968: 124 s. 
Tipos de aguas minero-medicinales ...

Hochscheid ${ }^{8}$. Igualmente, queda documentada la utilización en la Península Ibérica durante la Antigüedad de una gran variedad de aguas, ya que en la muestra estudiada están representados todos los principales tipos de aguas minero-medicinales existentes, aunque hay que tener en cuenta que en algunos casos no ha sido posible determinar el tipo exacto de agua, por lo que, en consecuencia, no se han podido incluir en el estudio de conjunto.

Pero la clasificación por el componente predominante del agua nos indica, además, una serie de interesantes preferencias. En su mayoría, los yacimientos se asocian a aguas sulfuradas $(25=55,5$ por 100$)$, seguidas, en orden decreciente, de bicarbonatadas $(10=22,2$ por 100$)$, cloruradas $(4=8,9$ por 100$)$, sulfatadas $(3=6,6$ por 100$)$ y, finalmente, de ferruginosas $(2=4,4$ por 100$)$. Las radioactivas, oligominerales y carbogaseosas, propiedades que se han considerado al margen de las anteriores, suponen, respectivamente, $13(=28,9$ por 100$), 9(=20$ por 100$)$ y 1 $(=2,2$ por 100$)$.

El análisis de conjunto nos da a conocer la aparente existencia de preferencias en la Antigüedad por determinados tipos de agua, lo que permite deducir las posibles causas que les llevaron a la utilización de unos tipos de aguas más que de otros. Esta visión se podría matizar más si se valoraran otros componentes secundarios, que pueden añadir al agua otras propiedades de interés. Pero este aspecto, dada su complejidad, exigiría un complejo tratamiento estadístico que no ha parecido oportuno realizar dada la calidad de los datos disponibles, aunque si se debe tener presente que en la Antigüedad han podido apreciarse otras características específicas del agua no valoradas en este estudio.

Si comparamos los valores medios de las aguas minero-medicinales de la Península Ibérica, obtenidos de las diversas recopilaciones existentes utilizadas como referencia, con las proporciones encontradas asociadas a yacimientos arqueológicos, se observan significativas diferencias según los tipos de aguas que cabe interpretarlas como consecuencia de una selección intencionada.

Resulta evidente en la Antigüedad la preferencia por las aguas sulfuradas entre todos los tipos de aguas identificados en mayor proporción de lo que cabria esperar según su abundancia en la Peninsula Ibérica.

Las clasificaciones más recientes recogen que las aguas sulfuradas en la Península lbérica apenas alcanzan el 25 por 100 del total de las

\footnotetext{
8 Weisgerber, G. 1975.
} 
aguas minero-medicinales (25 a 26 por 100), mientras que entre los yacimientos termales arqueológicos, clasificados según el mismo sistema, superan el 50 por 100 (55,5 por 100 exactamente). Esta proporción supone casi el doble de la teóricamente esperada, lo que no puede considerarse como efecto del azar ni de las características de la muestra.

Si empleamos un análisis estadístico «de hipótesis nula» entre las aguas sulfuradas de toda la Península Ibérica y las asociadas a yacimientos arqueológicos, se deduce que existe una probabilidad superior al 99 por 100 de que este hecho no se deba a circunstancias aleatorias.

Por el contrario, otros tipos de agua aparecen en proporción semejante a la que sería lógico encontrar dada su frecuencia real. Entre éstas cabe incluir las bicarbonatadas, oligominerales y radioactivas, por lo que se puede considerar que, aunque fueron utilizadas, no fueron buscadas con especial interés.

Otro conjunto de aguas estaria formado por aquellos tipos de agua que resultan asociados a yacimientos arqueológicos en menor frecuencia de la que, teóricamente, sería lógico esperar: serían las aguas cloruradas y, especialmente, las sulfatadas, carbogaseosas y ferruginosas.

Las aguas cloruradas sólo representan el 8,9 por 100 en yacimientos arqueológicos, aunque en las diversas recopilaciones su frecuencia varía entre el 16,9 y el 28,4 por 100. El hecho es también evidente en lo que respecta a las aguas ferruginosas, que sólo suponen el 4,4 por 100 de las aguas asociadas a yacimientos arqueológicos, proporción inferior a la real entre las agua minero-medicinales, ya que oscilan entre un 6 y un 20 por 100. Las sulfatadas y carbogaseosas aparecen en proporción menor a la mayoría de las recopilaciones existentes, especialmente las más actuales, pero su proporción ya se aproxima a la dada por alguna de ellas.

De estas sencillas observaciones se deduce la marcada preferencia por las aguas sulfuradas, una proporción semejante a la esperada en las bicarbonatadas, oligominerales y radioactivas y un uso menor del que cabría suponer en el caso de las aguas sulfatadas, cloruradas, oligominerales, como también al parecer, de las carbogaseosas y ferruginosas.

En cuanto a la temperatura de estas aguas, como ya se ha dicho, se han documentado 22 casos de aguas hipertermales, con temperaturas superiores a $36^{\circ} \mathrm{C}$; otros 17 casos eran aguas templadas, que oscilaban entre 30 a $33^{\circ} \mathrm{C}$, y finalmente, ocho casos eran frías, menores de $30{ }^{\circ} \mathrm{C}$.

Si en la Península Ibérica comparamos las temperaturas de las aguas documentadas en los yacimientos arqueológicos con una muestra de aguas minero-medicinales a nivel general se hace patente una marcada preferencia por las aguas de mayor temperatura. Las aguas frías apare- 
cen utilizadas en una proporción escasa, que oscila entre el 17 por 100 para las menores de $20{ }^{\circ} \mathrm{C}$ y el 26 por 100 para las de 20 a $30^{\circ} \mathrm{C}$. Esta proporción prácticamente se dobla en las aguas cuyas temperaturas están situadas entre 30 y $60^{\circ} \mathrm{C}$. Pero lo más significativo es que se utilizan más del 80 por 100 de las aguas de temperaturas superiores a $60^{\circ} \mathrm{C}$, alcanzando el 100 por 100 en las temperaturas aún más elevada.

Esta marcada preferencia permite suponer que la temperatura debió haber sido considerada como una de las caracteristicas principales a la hora de aplicar sus cualidades terapéuticas.

Estos hechos plantean la interesante cuestión de intentar esclarecer las posibles razones de las aparentes preferencias por determinados tipos de agua.

Del conjunto de estos análisis se deduce el conocimiento, aunque fuera empirico, de las características y propiedades de algunas aguas minero-medicinales, ya que de otro modo no se explican las preferencias señaladas. En consecuencia, cabe igualmente suponer la existencia de que poseian ciertas nociones sobre la adecuación de ciertos tipos de aguas para tratar determinadas afecciones.

\section{APÉNDICE I}

YACIMIENTOS ARQUEOLÓGICOS RELACIONADOS CON AGUAS MINEROMEDICINALES

\begin{tabular}{ccc} 
NOMBRE & TIPO AGUA & TEMPER \\
\hline
\end{tabular}

$\begin{array}{ll}\text { 1. Alange } \text { (Badajoz) }^{9} & \begin{array}{l}\text { Bicarbonatadas, cálcicas, radioacti- } 28{ }^{\circ} \mathrm{C} \\ \text { vas }\end{array}\end{array}$

2. Alhama de Aragón Bicarbonatadas, cálcicas, litínico- $32-37^{\circ} \mathrm{C}{ }^{11}$ (Alhama de Aragón, magnésicas, radioactivas

Zaragoza) ${ }^{12}$

${ }^{9}$ MORA, G. 1981: n. 81.

10 Armijo, M. de 1967: 390.

1 Armijo, M. de 1967: 390.

${ }^{12}$ Mora, G. 1981: n. ${ }^{\circ} 71$. 


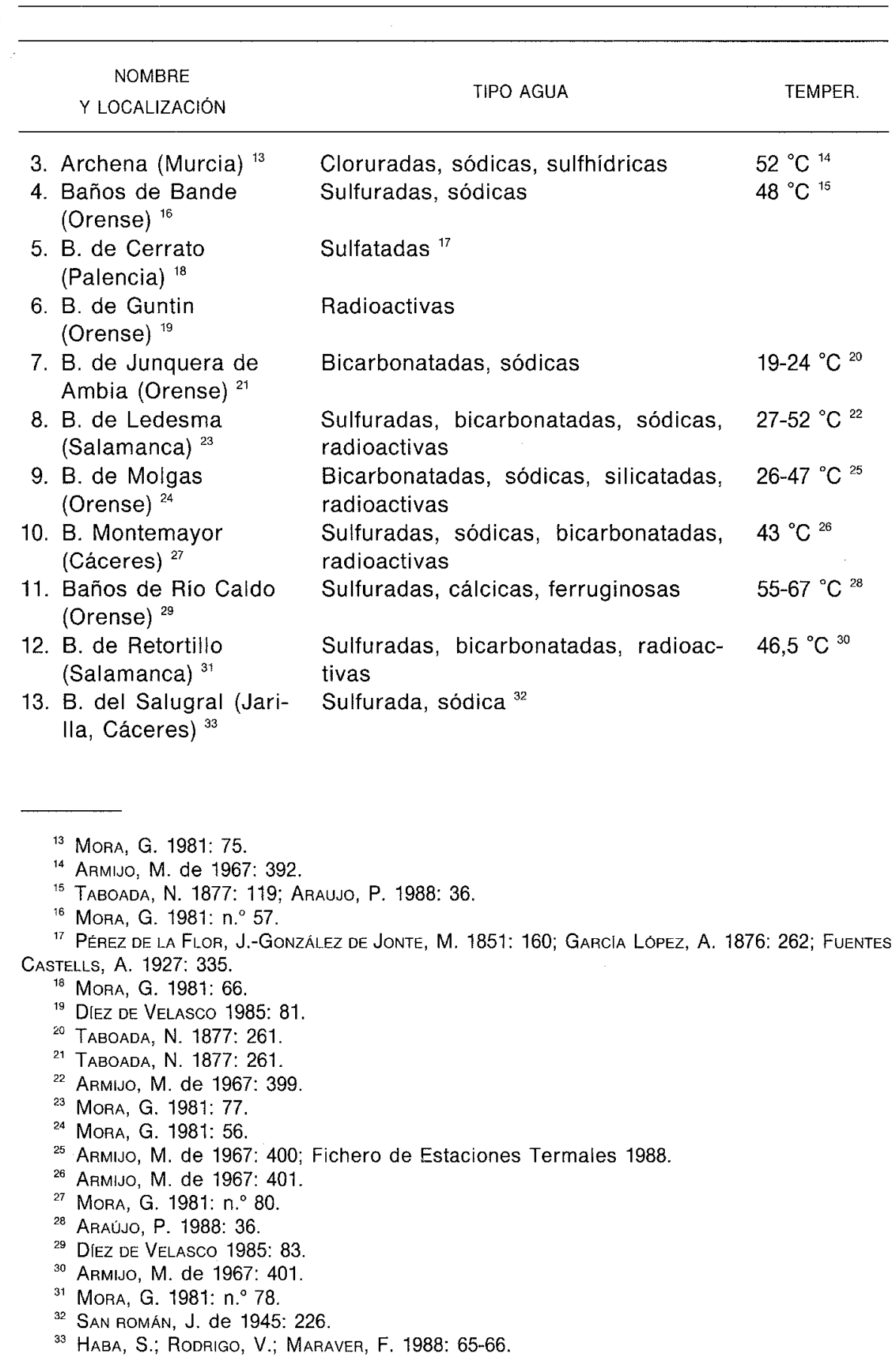




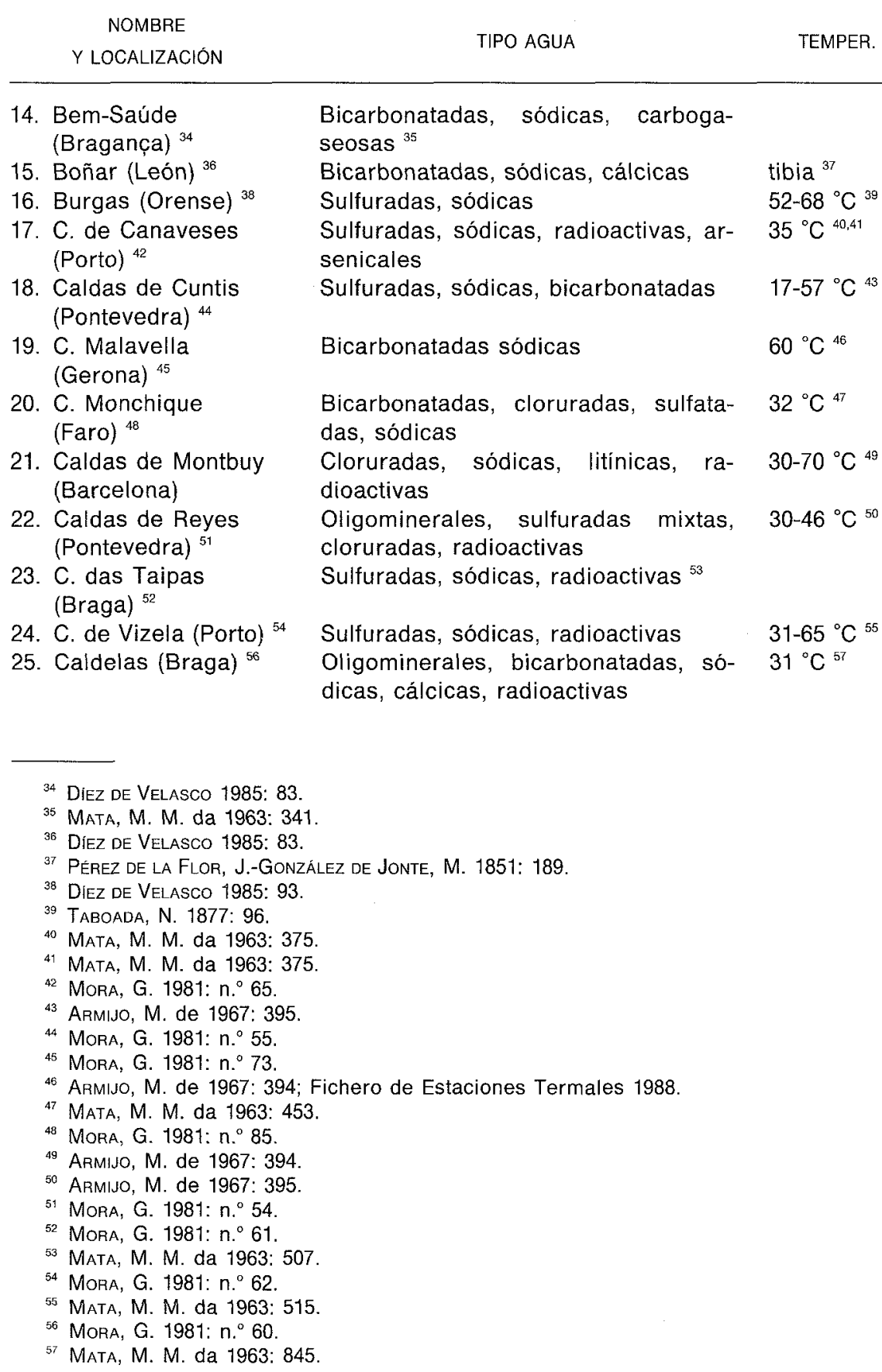

14. Bem-Saúde (Bragança) ${ }^{34}$

15. Boñar (León) ${ }^{36}$

16. Burgas (Orense) ${ }^{38}$

17. C. de Canaveses (Porto) ${ }^{42}$

18. Caldas de Cuntis (Pontevedra) ${ }^{44}$

19. C. Malavella (Gerona) ${ }^{45}$

20. C. Monchique (Faro) ${ }^{48}$

21. Caldas de Montbuy (Barcelona)

22. Caldas de Reyes (Pontevedra) ${ }^{51}$

23. C. das Taipas (Braga) ${ }^{52}$

24. C. de Vizela (Porto) ${ }^{54}$

25. Caldelas (Braga) ${ }^{56}$

Bicarbonatadas, sódicas, carbogaseosas ${ }^{35}$

Bicarbonatadas, sódicas, cálcicas tibia ${ }^{37}$

Sulfuradas, sódicas

Sulfuradas, sódicas, radioactivas, arsenicales

Sulfuradas, sódicas, bicarbonatadas $\quad 17-57^{\circ} \mathrm{C}$

Bicarbonatadas sódicas $\quad 60{ }^{\circ} \mathrm{C}{ }^{46}$

Bicarbonatadas, cloruradas, sulfata- $32{ }^{\circ} \mathrm{C}{ }^{47}$ das, sódicas

Cloruradas, sódicas, litínicas, ra- $30-70{ }^{\circ} \mathrm{C} 49$ dioactivas

Oligominerales, sulfuradas mixtas, $\quad 30-46{ }^{\circ} \mathrm{C}{ }^{50}$ cloruradas, radioactivas

Sulfuradas, sódicas, radioactivas ${ }^{53}$

Sulfuradas, sódicas, radioactivas

$31-65{ }^{\circ} \mathrm{C}^{55}$

Oligominerales, bicarbonatadas, só-

$31{ }^{\circ} \mathrm{C}^{57}$

dicas, cálcicas, radioactivas

${ }^{34}$ DiEZ DE VELASCO 1985: 83.

35 Mata, M. M. da 1963: 341.

${ }^{36}$ Diez de Velasco 1985: 83.

37 Pérez de la Flor, J.-González de Jonte, M. 1851: 189.

38 Diez de Velasco 1985: 93.

39 TABOADA, N. 1877: 96.

${ }^{40}$ Mata, M. M. da 1963: 375.

${ }^{41}$ Mata, M. M. da 1963: 375.

${ }^{42}$ Mora, G. 1981: n. 65.

${ }^{43}$ Armijo, M. de 1967: 395.

${ }^{44}$ Mora, G. 1981: n. ${ }^{\circ} 55$.

45 Mora, G. 1981: n. ${ }^{\circ} 73$.

46 Armiso, M. de 1967: 394; Fichero de Estaciones Termales 1988

${ }^{47}$ Mata, M. M. da 1963: 453.

48 Mora, G. 1981: n. 85.

49 Armijo, M. de 1967: 394.

50 ARMiJo, M. de 1967: 395.

51 MORA, G. 1981: n. ${ }^{\circ} 54$.

52 Mora, G. 1981: n. ${ }^{\circ} 61$.

${ }^{53}$ Mata, M. M. da 1963: 507.

${ }^{54}$ MoRA, G. 1981: $n .^{\circ} 62$.

55 Mata, M. M. da 1963: 515.

${ }^{56}$ Mora, G. 1981: n. ${ }^{\circ} 60$.

${ }^{57}$ Mata, M. M. da 1963: 845. 
26. Caminha (Viana do

Castelo) ${ }^{58}$

27. Carballo (La Coruña) ${ }^{59}$

Sulfuradas, cálcicas, cloruradas, só- $\quad 23-35^{\circ} \mathrm{C}{ }^{60}$ dicas

28. S. Vicente Pinheiro (Porto) ${ }^{63}$

29. Cerro de los Santos (Laguna de La Higuera, Albacete) ${ }^{65}$

30. Chaves (Vila Real) ${ }^{66}$

31. Fadagosa (Portalegre) ${ }^{68}$

32. Guitiriz (Lugo) ${ }^{70}$

33. Fitero (Navarra) ${ }^{72}$

34. Fuente Mortera (S. Juan de Tremañes, Gijón) ${ }^{74}$

35. Cortijo Ahorcado (Baeza, Jaén) ${ }^{75}$

36. La Garriga (Barcelona) $^{76}$
Sulfuradas, sódicas, radioactivas $18^{\circ} \mathrm{C}^{61,62}$

Sulfatadas, magnésicas, sódicas ${ }^{64}$

Bicarbonatadas, cálcicas, cloruradas Sulfuradas, sódicas ${ }^{69}$

Sulfuradas, fluoradas, sódicas Cloruradas, sódicas, sulfuradas, radioctivas

$73,5{ }^{\circ} \mathrm{C}^{67}$ $15{ }^{\circ} \mathrm{C}^{71}$ $47,5^{\circ} \mathrm{C}^{73}$ Clorurada, sódica $32-40{ }^{\circ} \mathrm{C}^{77}$

57 Mata, M. M. da 1963: 845.

58 Mora, G. 1981: n. 58.

59 Mora, G. 1981: n. 52.

60 Armijo, M. de 1967: 396.

${ }^{61}$ Mata, M. M. da 1963.

${ }^{62}$ MatA, M. M. da 1963: 903.

${ }^{63}$ MorA, G. 1981: n. ${ }^{\circ} 63$.

${ }^{64}$ Garcia López, A. 1876: 277; Fuentes Castells, A. 1927: 106.

${ }^{65}$ Ruiz Bremón, M.

${ }^{66}$ MORA, G. 1981: n. 59.

${ }^{67}$ MATA, M. M. da 1963: 395.

E8 MorA, G. 1981: n. 83.

69. MATA, M. M. da 1963: 601.

${ }^{70}$ Díez de Velasco 1985: 90.

${ }^{71}$ Armiso, M. de 1967: 398; Fichero de Estaciones Termales 1988.

${ }^{72}$ Mora, G. 1981: n. 70.

${ }^{73}$ Armilo, M. de 1967: 397.

${ }^{74}$ Mora, G. 1981: n. 65.

${ }^{75}$ Mora, G. 1981: n. ${ }^{\circ} 86$.

76 MORA, G. 1981.

${ }^{77}$ Garcia Lopez, A. 1876: 230. 
Tipos de aguas minero-medicinales ...

\begin{tabular}{|c|c|c|}
\hline $\begin{array}{c}\text { NOMBRE } \\
\text { Y LOCAL.IZACIÓN }\end{array}$ & TIPO AGUA & TEMPER. \\
\hline 37. Linares (Segovia) ${ }^{78}$ & Sulfatadas, sódicas & $21^{\circ} \mathrm{C}^{79}$ \\
\hline 38. Lisboa ${ }^{80}$ & Sulfuradas, sódicas & $25-37^{\circ} \mathrm{C}^{81}$ \\
\hline 39. Lugo ${ }^{82}$ & Sulfuradas, sódicas, bicarbonatadas & $30-43^{\circ} \mathrm{C}^{83}$ \\
\hline $\begin{array}{l}\text { 40. } \text { Mondariz } \\
\text { (Pontevedra) }^{84}\end{array}$ & $\begin{array}{l}\text { Bicarbonatadas, sódicas, carbogaseo- } \\
\text { sas }\end{array}$ & $13-16{ }^{\circ} \mathrm{C}^{85}$ \\
\hline 41. Ontaneda (Santander) & Sulfurada, cálcica & \\
\hline 42. Panticosa (Huesca) ${ }^{86}$ & $\begin{array}{l}\text { Oligominerales, sulfuradas, silicata- } \\
\text { das, radioactivas }\end{array}$ & $27-51^{\circ} \mathrm{C}^{87}$ \\
\hline $\begin{array}{l}\text { 43. S. Pedro Sul } \\
\text { (Viseu) }\end{array}$ & Sulfuradas, sódicas, radioactivas & $68^{\circ} \mathrm{C}^{89}$ \\
\hline $\begin{array}{l}\text { 44. San Gregorio de Bro- } \\
\text { zas (Cáceres) }{ }^{90}\end{array}$ & Sulfuradas, sódicas & $19^{\circ} \mathrm{C}^{91}$ \\
\hline $\begin{array}{l}\text { 45. Santa Cruz del Loyo } \\
\text { (Paradela, Lugo) }\end{array}$ & Ferruginosas ${ }^{32}$ & \\
\hline $\begin{array}{l}\text { 46. Santa Eulalia de Cal- } \\
\text { delas (Pontevedra) }\end{array}$ & Sulfuradas, sódicas ${ }^{94}$ & \\
\hline 47. Saelices (Cuenca) & Ferruginosas, magnésicas & Fría ${ }^{\circ} \mathrm{C}^{96}$ \\
\hline $\begin{array}{l}\text { 48. Senhora da Luz } \\
\text { (Faro })^{97}\end{array}$ & Bicarbonatadas, cálcicas ${ }^{98}$ & \\
\hline 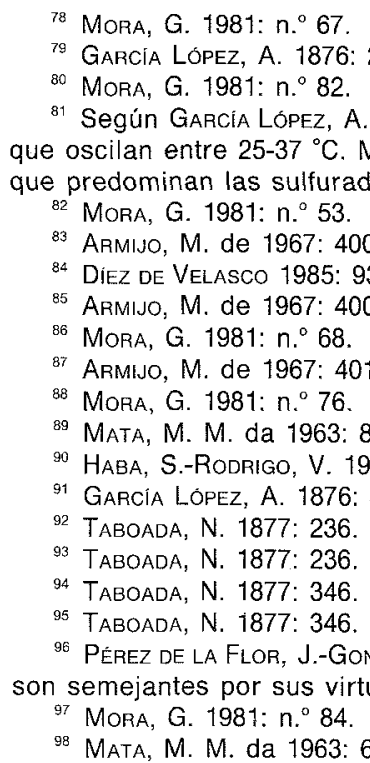 & $\begin{array}{l}78 . \\
\text { 1876: 294, Lisboa tiene muchas fuentes term } \\
\text { TA, M. M. da } 1963, \text { recoge también diversas } \\
\text { \$. } \\
\text { Fichero de Estaciones Termales } 1988 . \\
1 . \\
1: \text { 17. } \\
02 . \\
\text { ÁLEZ DE JONTE, M. } 1851 \text { : } 494 \text {. Según estos aut } \\
\text { 1es a las de Solán de Cabras (Beteta, Cuen } \\
\end{array}$ & $\begin{array}{l}\text { es «sulfurosas», } \\
\text { uentes entre las }\end{array}$ \\
\hline
\end{tabular}


LUCIA MOLTÓ

NOMBRE

Y LOCALIZACIÓN

TIPO AGUA

TEMPER.

49. Tejares

(Salamanca) $^{99}$

50. Tiermas (Zaragoza) ${ }^{100}$

Sulfuradas, sódicas, cloruradas

$33{ }^{\circ} \mathrm{C}^{101}$

51. Torreblanca (Fuengi- Sulfuradas, sódica

Frias rola, Málaga) ${ }^{102}$

52. Villavieja (Vilavella Sulfatadas cálcicas

$29-45{ }^{\circ} \mathrm{C}^{103}$ de Nules,

Castellón) ${ }^{104}$

99 Mora, G. 1981: n. 79

100 Mora, G. 1981: n. 69.

101 Pérez de la Flor, J., y González de Jonte, M.: 516.

102 Mora, G. 1981: n. 87.

103 ArmiJo, M. de 1967: 403; Fichero de Estaciones Termales 1988.

104 MorA, G. 1981: n. 74. 
Tipos de aguas minero-medicinales ...

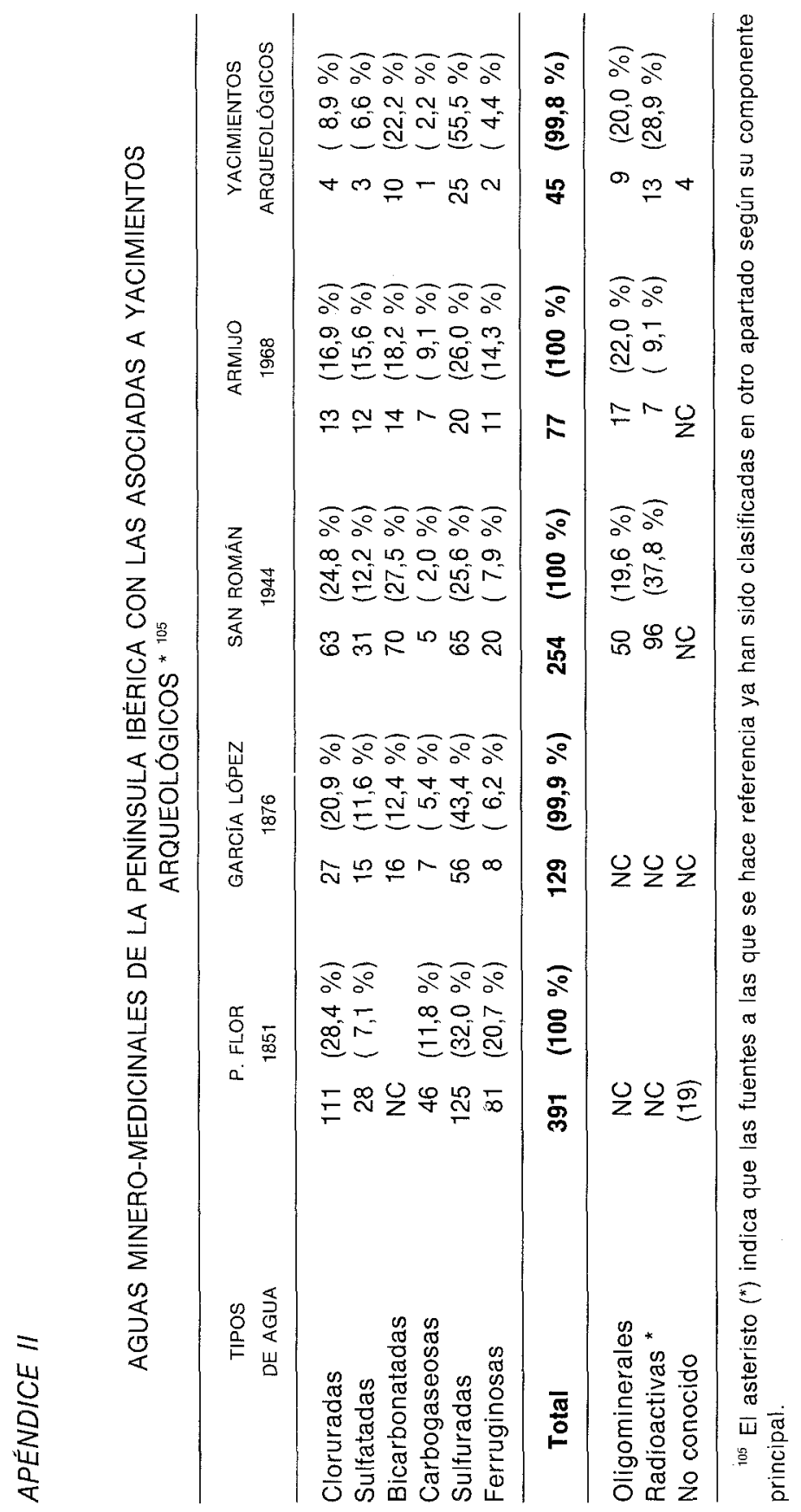




\section{RESUMEN}

Las aguas de algunos yacimientos termales que ofrecen restos arqueológicos y que están relacionados con balnearios actuales o en desuso han sido recogidas con objeto de iniciar el estudio de los tipos de aguas utilizadas en la Antigüedad.

El hecho más destacado es el claro predominio en estos yacimientos de aguas sulfuradas e hipertermales.

\section{ABSTRACT}

The thermal waters found in connection with archaeological setlements and related with present or past documented thermal baths have been gathered in order to study the types of thermal water used in Antiquity.

The most interesting result is the marked preference for sulfuric and hiperthermal waters. 